\title{
Amphoteric Surface Active Agents with Heterocyclic Ring Based on Industrial Wastes
}

\author{
W. I. A. El-Dougdoug ${ }^{\text {a,b }}$ and K. A. H. Hebash ${ }^{\text {a }}$ \\ ${ }^{a}$ Chemistry Department, Faculty of Science, Benha University, \\ Benha, Egypt and ${ }^{b}$ Chemistry Department, Universal College, \\ Umm Al-Qura University, Makkah El-Mukarramah, Saudi \\ Arabia.
}

\begin{abstract}
Q ERIES of surface active agents as amphoteric surfactants $\checkmark$ were prepared by the reaction of $\mathrm{N}$-acyl-2-aminothiazole and/or $\mathrm{N}$-acyl-2-aminopyridine of pure [(decanoic C10 : 0 , dodecanoic C12: 0, tetradecanoic C14 : 0, hexadecanoic C16:0, octdecanoic C18:0, octdec 9-enoic C18 : 1, octdec 9, 12-dienoic C18 : 2, mixed fatty acids of Gawafa seed fat and mixed fatty acids of Grape seed oil (GSO)] with 3-chloro 2-hydroxypropane sulfonate and chloroacetic acid to produce amido-sulfobetaine [ IVa-i and VI a-i] and amido-betaine [Va-i and VIIa-i] with heterocyclic ring, respectively. The structure of the prepared surface active agents were confirmed by microanalysis, IR and ${ }^{1} \mathrm{H}-\mathrm{NMR}$ spectra. The prepared surface active agents have a double properties, antimicrobial and high emulsification power.

Keywords: Gawafa seed fat (GSF), Grape seed oil (GSO), Amphoteric surfactants antimicrobial and Surface active properties.
\end{abstract}

The surface active agents derived from natural sources acquired more valuable interest from two points of view; economic (has low price) and environmental pollution (reduced pollution). Our interest was extended to prepare the surface active agents from rubbish sources like fatty acids were extracted from both Mangifera indica ${ }^{(1-2)}$, rice bran oil ${ }^{(3)}$ and Gawafa fat ${ }^{(4)}$. Since Gawafa seeds have good percentage of saturated fatty acids especially octanoic, dodecanoic, hexadecanoic and octadecanoic acids and small percentage of decanoic and tetradecanoic acids. Also, Grape seed oil has small percentage of saturated fatty acids especially, hexadecanoic and octadecanoic acids and good percentage of unsaturated fatty acids especially linoleic (C18:2) and oleic (C18:1) acids. In general, grape seed oil is very similar to sunflower oil, it is a good all-round oil, high in essentional fatty acid linoleic, but very low in easily oxidized linolenic acid ${ }^{(5)}$; it is also, low in saturates. It was interesting to prepare some biologically active simple heterocycles which constitute an important class of organic compounds with diverse biological activities ${ }^{(6-9)}$. Numerous fatty 
alcohols are now more available in their pure form and inexpensive enough to provide the chemical field with a wealth of reactions in which fatty alcohols are used as raw material in a variety of industrial products like pharmaceuticals ${ }^{(10-11)}$, cosmetics, surfactants, paints,... etc. The amphoteric surface active agents prepared by using 2-hydroxypropane sulfonate (sulfobetaine) offer the most promise as dispersant in the soap based detergents. In the extension of our interest in the preparation and study of the surface properties of surface active agents derived from natural source with heterocyclic ring, we tried to prepare the amidosulfobetaine and amidobetaine amphoteric surface active agents with simple heterocyclic ring from commercial sources (c.f. Scheme 1 ) and study their double properties, antimicrobial and surface active agents properties.
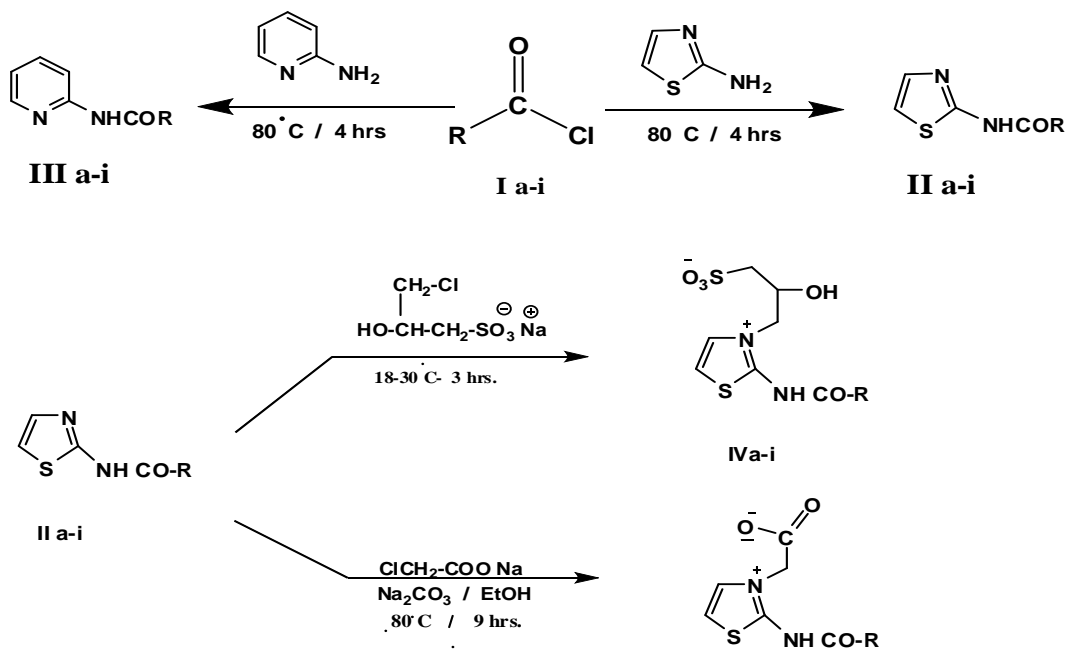

V a-i
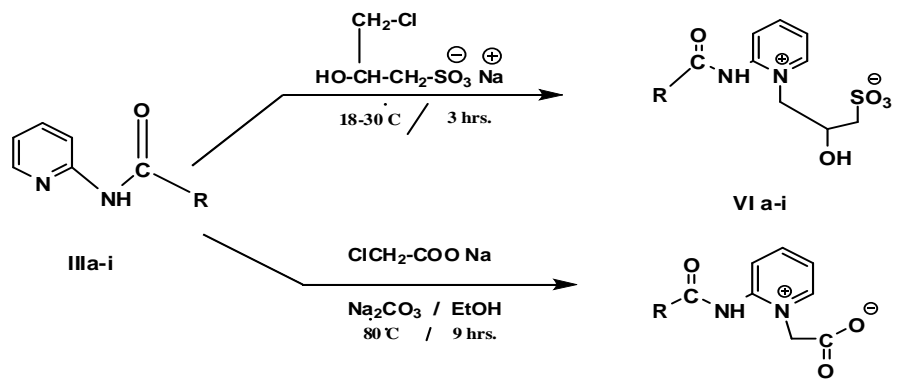

VII a-i

$\mathbf{R}=, \mathrm{CH}_{3}-\left(\mathrm{CH}_{2}\right)_{8}-, \mathrm{CH}_{3}-\left(\mathrm{CH}_{2}\right)_{10}-, \mathrm{CH}_{3}-\left(\mathrm{CH}_{2}\right)_{12}-, \mathrm{CH}_{3}-\left(\mathrm{CH}_{2}\right)_{14}-, \mathrm{CH}_{3}-\left(\mathrm{CH}_{2}\right)_{16}-, \mathrm{CH}_{3}-\left(\mathrm{CH}_{2}\right)_{7} \mathrm{CH}=\mathrm{CH}-\left(\mathrm{CH}_{2}\right)_{7^{-}}, \mathrm{CH}_{3}-\left(\mathrm{CH}_{2}\right)_{4}$ $\mathrm{CH}=\mathrm{CH}-\mathrm{CH}_{2} \mathrm{CH}=\mathrm{CH}-\left(\mathrm{CH}_{2}\right)_{7}^{-}$, mixed alkyl chain of Gawafa fat, mixed alkyl chain of Grape oil. crossponding to $a, b, c, d, e, f, g, h$, and $i$

\section{Scheme 1}

Egypt. J. Chem. 54, No. 2 (2011) 


\section{Experimental}

The IR spectrum was measured by Pye-Unicam SR-1000 infra-red Spectrophotometer as $\mathrm{KBr}$ disk or nujl mull and ${ }^{1} \mathrm{H}-\mathrm{NMR}$ was done in DMSO and /or $\mathrm{CDCl}_{3}$ as solvent and tetramethylsilane (TMS) as internal standard [Varian EM-390] Spectrophotometer operating at $260 \mathrm{MHz}$.

Industrial wastes of Gawafa seeds

Grape seeds, were kindly supplied by El-Nasr Company of canned products, Kaha, and grape seed from Aga juices company Egypt. The oil was extracted from the seeds. The specifications are given in Table 1.

Hydrolysis of crude Gawafa seeds fat and or grape seed oil

The procedure described by El-Sawy et al. ${ }^{(12)}$ was followed. The fatty acids mixture was analyzed by G. L. C. and their compositions are given in Table 1

TABLE 1. Fatty acids composition and chemical characteristic of Gawafa fat and Grape oil $^{(12)}$.

\begin{tabular}{|c|c|c|}
\hline \multirow{2}{*}{$\begin{array}{c}\text { Chemical } \\
\text { characteristics }\end{array}$} & \multicolumn{2}{|c|}{$\frac{\text { Fatty acid composition }}{\text { Peak area } \%}$} \\
\hline & Gawafa fat & Grape oil \\
\hline Gawafa fat & Saturated fatty acids: & Saturated fatty acids: \\
\hline A.V. $=25.5$ & $\begin{array}{lll}\text { Caprylic acid } & \mathrm{C}_{8: 0} & 12.51\end{array}$ & Lauric acid $\quad \mathrm{C}_{12: 0} \quad 00.61$ \\
\hline $\mathrm{V} .=11.8$ & Capric acid $\quad \mathrm{C}_{10: 0}^{0.0} \quad 01.94$ & Myristic acid $\quad \mathrm{C}_{14: 0} \quad 00.30$ \\
\hline S. V. $=197.8$ & $\mathrm{C}_{12: 0} \quad 26.61$ & Palmitic acid $\mathrm{C}_{16: 0} \quad 10.23$ \\
\hline Unsap $=2.03$ & $\begin{array}{lll}\text { Myristic acid } & C_{14: 0} & 03.25 \\
\text { Palmitic acid } & C_{16.0} & 20.25\end{array}$ & Stearic acid \\
\hline Grape Oil:- & $\mathrm{C}_{18: 0}^{10.0} 31.00$ & Unsaturated Fatty acids: \\
\hline$\overline{\mathrm{A} . \mathrm{V} .}=3.34$ & Unsaturated Fatty acids: & Palmitoleic acid $\mathrm{C}_{16.1} \quad 3.10$ \\
\hline I.V. $=132.8$ & $\mathrm{C}_{18: 1} \quad 04.44$ & Oleic acid $\quad \mathrm{C}_{18: 1} 16.21$ \\
\hline S. N. $=187.6$ & & Linoleic acid \\
\hline Unsap $=2.83$ & & Linolenic acid $\quad \mathrm{C}_{18: 3} \quad 1.23$ \\
\hline
\end{tabular}

\section{Preparation of acyl chloride}

It was prepared as vicous oil from the corresponding acids (decanoic, dodecanoic, tetradecanoic, hexadecanoic, octadecanoic, octadec9-enoic, octadec 9,12 dienoic acid, mixed fatty acids of gawafa fat and mixed fatty acids of grape seed oil, respectively according to Youngs et al. methods ${ }^{(13)}$.

\section{Preparation of amido derivatives ${ }^{(6)}$}

$\mathrm{N}$-Acyl-2-aminopyridine and $\mathrm{N}$-acyl-2-aminothiazole derivatives were prepared by condensation of equamolar amounts of the corresponding pure fatty acid chloride of (decanoic C10:0, dodecanoic C12:0, tetradecanoic C14:0, hexadecanoic C16:0, octadecanoic C18:0, 9-ctadecenoic C18:1 acids , mixed fatty acids extracted from 
Gawafa fat and /or Gape seed oil) with 2-aminothiazole and/or 2-aminopyridine, respectively. The reaction mixture was heated at $80{ }^{\circ} \mathrm{C}$ for $4 \mathrm{hr}$ in the presence of catalytic amount of pipridine and dry benzene as solvent. The organic solvent was removed under reduced pressure, and residue was recrystallized from isopropanol. The yield percentage was 84-95\%, the structures of compounds IIa-i, IIIa-i, was confirmed by IR and $\mathrm{H}^{1} \mathrm{NMR}$ (c.f. Table 2).

Preparation of sodium 1-chloro-2-hydroxy-3-propanesulfonate ${ }^{(6)}$

Epichlorohydrine (50 g., $0.45 \mathrm{~mol}$ ) was gradually added to sodium bisulfate $(64.7 \mathrm{~g} ., 0.621 \mathrm{~mol})$ and sodium sulfate $(25.0 \mathrm{~g} ., 0.199 \mathrm{~mol})$ dissolved in $130 \mathrm{ml}$ water. The reaction temperature was maintained between $18-30{ }^{\circ} \mathrm{C}$ with aid of a cooling ice bath due to the exothermal reaction. The reaction product precipitated gradually and the $\mathrm{pH}$ of the solution remained at 6 . After agitation for $2 \mathrm{hr}$ at room temperature, the reaction product was removed by filtration and dried. The yield percentage was $96 \%$ and used as crude products in the next steps.

Preparation of amido sulfobetaines $(I V, V I) . a-l^{(6)}$

They are prepared by placing 0.02 mol of sodium 1-chloro-2-hydroxy- 3propylene sulfonate in $45 \mathrm{ml}$ of water and $0.02 \mathrm{~mol}$ of IIa-l or IIIa-l (derivatives) dissolved in $100 \mathrm{ml}$ of ethanol, and refluxed for $3 \mathrm{hr}$. The reaction mixture was cooled to $50^{\circ} \mathrm{C}$, then $0.03 \mathrm{~mol}$ of sodium carbonate was added with refluxing for $6 \mathrm{hr}$. After cooling the product was filtered to remove the residual solid and extracted with $200 \mathrm{ml}$ of petroleum-ether $\left(40-60^{\circ} \mathrm{C}\right)$. The remainder (water and alcohol phase) was evaporated to dryness, washed with mixed solvents dichloroethane and acetone (v/v 50\%) and dissolved in 75\% ethanol. Finally, the pure product was obtained in 55-64\% yield. The structure and purity were identified by TLC, elemental analysis, IR and $\mathrm{H}^{1} \mathrm{NMR}$. (c.f. Table 2).

\section{Preparation of amidobetaines $(V, V I I) a-l^{(6)}$}

They were prepared by refluxing a solution of 0.016 mol of IIa-i or IIIa-i in $100 \mathrm{ml}$ of absolute ethyl alcohol and $140 \mathrm{ml}$ of aqueous solution containing 40 grams of sodium chloroacetate and 5 grams of sodium bicarbonate in three necked flask with stirring at $80^{\circ} \mathrm{C}$ for $9 \mathrm{hr}$. After cooling and removing the solvent to dryness, the reminder was dissolved in distilled water and filtered to remove the insoluble material. The filtrate was evaporated to remove water, dissolved in absolute ethyl alcohol and then filtered to remove inorganic salts (three times). The final products were identified by elemental analysis, IR and ${ }^{1} \mathrm{H}-\mathrm{NMR}$. The yield percentage was $(80-90 \%)($ c. f. Table 2).

\section{Evaluation method of surface active properties}

Surface and interfacial tension measurements

Surface and interfacial tension measurements of the prepared surfactants were made at room temperature $\left(25^{\circ} \mathrm{C}\right)$ with a Du Nouy tensiometer (DST 30 Series) using distilled water solution of $0.1 \%$ weight concentration ${ }^{(14)}$. The surface tension of the used distilled water was $73 \mathrm{mN} / \mathrm{m}$ and the interfacial tension between medicinal paraffin oil and distilled water was $56.2 \mathrm{mN} / \mathrm{m}$. Surfactant solutions were aged for

Egypt. J. Chem. 54, No. 2 (2011) 
$1 / 2 \mathrm{hr}$ before any measurements were made. Three readings were made on each sample to determine any change with time and to obtain an average value ${ }^{(10)}$.

Kraft point was measured as the temperature at which $1 \%$ dispersion solution becomes clear on gradual heating ${ }^{(15)}$.

Wetting time was determined by immersing a sample of cotton fabric in $0.1 \%$ aqueous solution of the surfactants ${ }^{(16)}$.

Foaming properties were measured according to Ross and Miles method ${ }^{(17)}$. The foam production for $0.1 \%$ solution was measured by the foam height initially produced.

Emulsion power: the emulsion was prepared from $10 \mathrm{ml}$. of $20 \mathrm{~m}$ mol aqueous solution of surfactant and $5 \mathrm{ml}$ of paraffin oil at $25^{\circ} \mathrm{C}$. The emulsifying property was determined by the ratio between the oil volume separating from the emulsion layer ${ }^{(18)}$.

Stability to hydrolysis: A mixture of $10 \mathrm{~m}$ mol surfactant and $10 \mathrm{ml} .0 .05 \mathrm{~N} \mathrm{NaOH}$ were placed in a thermostat at $40^{\circ} \mathrm{C}$. The time required for a sample solution to be clouded as a result of hydrolysis shows the stability of surfactant to hydrolysis ${ }^{(19)}$.

Hydrophilic-lipophilic balance (HLB) of a surfactant is a measure of the degree to which it is hydrophilic or lipophilic, determined by calculating values for the different regions of the molecule, as described ${ }^{(20,21)}$

Biodegradability \%: Biodegradation is carried out by bacteria in nature. By enzymatic reactions, a surfactant molecule is ultimately converted into carbon dioxide, water and oxides of the other elements. If the surfactant does not undergo natural biodegradation then it is stable and persists in the environment. For surfactants the rate of biodegradation varies from 1-2 hr for fatty acids, 1-2 days for linear alkyl benzene sulfonates and several months for branched alkyl benzene sulfonates. The rate of biodegradation depends on the surfactant concentration, $\mathrm{pH}$ and temperature. The temperature effect is particularly important, since the rate can vary by as much a factor of five between summer and winter in Northern Europe. Two criteria are important when testing for biodegradation: (1) Primary degradation that results in loss of surface activity. (2) Ultimate biodegradation, i.e. conversion into carbon dioxide, which can be measured using closed bottle tests. The rate of biodegradation also depends on the surfactant structure. For example, the surfactant must be water soluble. Biodegradability percentage was determined following the method of Eter ${ }^{(22)}$, according to the following equation:

where: $\quad \gamma \mathrm{t}=$ Surface tension at time $\mathrm{t}$.

$$
\left.\mathrm{D} \%=\frac{\gamma^{\mathbf{t}}-\gamma^{\mathbf{b}} \mathbf{t}-\gamma^{\mathbf{0}}}{[\mathrm{s}}\right] \times 100
$$

$\gamma 0=$ Surface tension at time zero

$\gamma \mathrm{bt}=$ Surface tension of blank at time $\mathrm{t}$, without sample.

Egypt. J. Chem. 54, No. 2 (2011) 


\section{Results and Discussion}

The preparation of amphoteric surfactants $[\mathrm{IV}-\mathrm{VII}]_{\mathrm{a}-\mathrm{i}}$ was performed according to the preparation methodology giving suitable yields. Where, the fatty acids converted to the corresponding fatty acid chloride according to Youngs et al. $\operatorname{method}^{(13)}$. Then, react with 2-aminothiazole and 2-aminopyridine to afford $\mathrm{N}$-acyl aminothiazole [IIa-i] and 2-N-acyl aminopyridine [IIIa-i], respectively. The IR spectra of the prepared amido-derivatives showed band at $\mathrm{cm}-1$ for $(v \mathrm{~N}-\mathrm{H})$, ( $\mathrm{vC}=\mathrm{O}$ of sec. amide). Micro-analysis; Infrared (IR) and Proton Nuclear Magnetic Resonance (1H-NMR) spectra; were carried out to establish the structure of some examples of the prepared amphoteric compounds (Tables $2 \& 3$ ).

TABLE 2. Micro-analytical data of the synthesized amphoteric sulfobetaine and betaine surfactants containing thiazolium or pyridinium moiety.

\begin{tabular}{|c|c|c|c|c|c|c|c|c|c|c|c|}
\hline \multirow{3}{*}{$\begin{array}{l}\text { Compound } \\
\text { number }\end{array}$} & \multirow{3}{*}{$\begin{array}{c}\text { Mol. } \\
\text { Form. }\end{array}$} & \multirow{3}{*}{$\begin{array}{l}\text { Mol. } \\
\text { wt. }\end{array}$} & \multirow{3}{*}{$\begin{array}{c}\text { Yield } \\
\%\end{array}$} & \multicolumn{8}{|c|}{ Microanalysis } \\
\hline & & & & \multicolumn{2}{|l|}{$\mathrm{C} \%$} & \multicolumn{2}{|l|}{$\mathbf{H \%}$} & \multicolumn{2}{|l|}{ N\% } & \multicolumn{2}{|l|}{ S\% } \\
\hline & & & & Calc. & Fd. & Calc. & Fd. & Calc. & Fd. & Calc. & Fd. \\
\hline IVa & $\mathrm{C}_{16} \mathrm{H}_{28} \mathrm{~N}_{2} \mathrm{~S}_{2} \mathrm{O}_{5}$ & 392.14 & 64 & 48.96 & 48.90 & 7.19 & 7.10 & 7.14 & 7.10 & 16.34 & 16.30 \\
\hline $\mathrm{IVb}$ & $\mathrm{C}_{18} \mathrm{H}_{32} \mathrm{~N}_{2} \mathrm{~S}_{2} \mathrm{O}_{5}$ & 420.18 & 62 & 51.40 & 51.30 & 7.67 & 7.62 & 6.66 & 6.60 & 15.25 & 15.20 \\
\hline IVc & $\mathrm{C}_{20} \mathrm{H}_{36} \mathrm{~N}_{2} \mathrm{~S}_{2} \mathrm{O}_{5}$ & 448.21 & 59 & 53.54 & 53.30 & 8.09 & 8.07 & 6.24 & 6.24 & 14.29 & 14.30 \\
\hline IVd & $\mathrm{C}_{22} \mathrm{H}_{40} \mathrm{~N}_{2} \mathrm{~S}_{2} \mathrm{O}_{5}$ & 476.24 & 55 & 55.43 & 55.40 & 8.46 & 8.45 & 5.88 & 5.80 & 13.45 & 13.40 \\
\hline IVe & $\mathrm{C}_{24} \mathrm{H}_{44} \mathrm{~N}_{2} \mathrm{~S}_{2} \mathrm{O}_{5}$ & 504.27 & 64 & 57.11 & 57.01 & 8.79 & 8.70 & 5.55 & 5.50 & 12.71 & 12.70 \\
\hline IVf & $\mathrm{C}_{24} \mathrm{H}_{42} \mathrm{~N}_{2} \mathrm{~S}_{2} \mathrm{O}_{5}$ & 502.25 & 58 & 57.34 & 57.30 & 8.42 & 8.30 & 5.57 & 5.50 & 12.76 & 12.70 \\
\hline IVg & $\mathrm{C}_{24} \mathrm{H}_{40} \mathrm{~N}_{2} \mathrm{~S}_{2} \mathrm{O}_{5}$ & 500.24 & 61 & 57.57 & 57.60 & 8.05 & 8.00 & 5.59 & 5.60 & 12.81 & 12.8 \\
\hline IVh & Mixed of GSF & - & 62 & - & - & - & - & - & - & - & $-*$ \\
\hline IVi & Mixed of G.O & - & 63 & - & - & - & - & - & - & - & $-*$ \\
\hline $\mathrm{Va}$ & $\mathrm{C}_{15} \mathrm{H}_{24} \mathrm{~N}_{2} \mathrm{SO}_{3}$ & 312.15 & 84 & 57.66 & 57.60 & 7.74 & 7.70 & 8.97 & 8.90 & 10.26 & 10.20 \\
\hline $\mathrm{Vb}$ & $\mathrm{C}_{17} \mathrm{H}_{28} \mathrm{~N}_{2} \mathrm{SO}_{3}$ & 340.18 & 88 & 59.97 & 59.90 & 8.29 & 8.20 & 8.23 & 8.20 & 9.42 & 9.42 \\
\hline $\mathrm{Vc}$ & $\mathrm{C}_{19} \mathrm{H}_{32} \mathrm{~N}_{2} \mathrm{SO}_{3}$ & 368.53 & 80 & 61.92 & 61.90 & 8.75 & 8.60 & 7.60 & 7.60 & 8.70 & 8.70 \\
\hline $\mathrm{Vd}$ & $\mathrm{C}_{21} \mathrm{H}_{36} \mathrm{~N}_{2} \mathrm{SO}_{3}$ & 396.25 & 90 & 63.60 & 63.30 & 9.15 & 9.20 & 7.06 & 7.05 & 8.09 & 8.00 \\
\hline $\mathrm{Ve}$ & $\mathrm{C}_{23} \mathrm{H}_{40} \mathrm{~N}_{2} \mathrm{SO}_{3}$ & 424.28 & 81 & 65.05 & 65.00 & 9.49 & 9.44 & 6.60 & 6.50 & 7.55 & 7.60 \\
\hline Vf & $\mathrm{C}_{23} \mathrm{H}_{38} \mathrm{~N}_{2} \mathrm{SO}_{3}$ & 422.26 & 83 & 65.36 & 65.30 & 9.06 & 9.06 & 6.63 & 6.60 & 7.59 & 7.50 \\
\hline $\mathrm{Vg}$ & $\mathrm{C}_{23} \mathrm{H}_{36} \mathrm{~N}_{2} \mathrm{SO}_{3}$ & 420.24 & 82 & 65.68 & 65.60 & 8.63 & 8.60 & 6.66 & 6.60 & 7.63 & 7.60 \\
\hline VIa & $\mathrm{C}_{18} \mathrm{H}_{30} \mathrm{~N}_{2} \mathrm{SO}_{5}$ & 386.19 & 55 & 55.94 & 55.80 & 7.82 & 7.80 & 7.25 & 7.30 & 8.30 & 8.20 \\
\hline $\mathrm{VIb}$ & $\mathrm{C}_{20} \mathrm{H}_{34} \mathrm{~N}_{2} \mathrm{SO}_{5}$ & 414.22 & 61 & 57.94 & 57.80 & 8.27 & 8.30 & 6.76 & 6.70 & 7.73 & 7.70 \\
\hline VIc & $\mathrm{C}_{22} \mathrm{H}_{38} \mathrm{~N}_{2} \mathrm{SO}_{5}$ & 442.25 & 56 & 59.70 & 59.62 & 8.65 & 8.70 & 6.33 & 6.30 & 7.24 & 7.30 \\
\hline VId & $\mathrm{C}_{24} \mathrm{H}_{30} \mathrm{~N}_{2} \mathrm{SO}_{5}$ & 470.67 & 59 & 61.24 & 61.22 & 8.99 & 8.80 & 5.95 & 5.90 & 6.81 & 6.80 \\
\hline VIe & $\mathrm{C}_{24} \mathrm{H}_{42} \mathrm{~N}_{2} \mathrm{SO}_{5}$ & 498.31 & 64 & 62.62 & 62.60 & 9.30 & 9.20 & 5.62 & 5.60 & 6.43 & 6.40 \\
\hline VIIc & $\mathrm{C}_{21} \mathrm{H}_{34} \mathrm{~N}_{2} \mathrm{O}_{3}$ & 362.51 & 90 & 69.58 & 69.50 & 9.45 & 9.40 & 7.73 & 7.70 & - & - \\
\hline VIId & $\mathrm{C}_{23} \mathrm{H}_{38} \mathrm{~N}_{2} \mathrm{O}_{3}$ & 390.56 & 86 & 70.73 & 70.70 & 9.81 & 9.70 & 7.17 & 7.10 & - & - \\
\hline VIIe & $\mathrm{C}_{25} \mathrm{H}_{42} \mathrm{~N}_{2} \mathrm{O}_{3}$ & 418.61 & 82 & 71.73 & 71.70 & 10.11 & 10.00 & 9.69 & 9.68 & - & - \\
\hline VIIf & $\mathrm{C}_{25} \mathrm{H}_{40} \mathrm{~N}_{2} \mathrm{O}_{3}$ & 416.60 & 85 & 72.08 & 72.00 & 9.68 & 9.60 & 6.72 & 6.72 & - & - \\
\hline VIIg & $\mathrm{C}_{25} \mathrm{H}_{38} \mathrm{~N}_{2} \mathrm{O}_{3}$ & 414.58 & 81 & 72.43 & 72.40 & 9.29 & 9.30 & 6.76 & 6.70 & - & - \\
\hline
\end{tabular}

* Mixed compounds.

Egypt. J. Chem. 54, No. 2 (2011) 
TABLE 3. Spectral data of some representing examples for amidothiazole and some synthesized amphoteric surfactants.

\begin{tabular}{|c|c|c|}
\hline $\begin{array}{l}\text { Compound } \\
\text { No. }\end{array}$ & ${ }^{1}$ HNMR $(\delta=$ ppm $)$ & $\operatorname{IR}\left(v / \mathrm{cm}^{-1}\right)$ \\
\hline $\mathbf{I I}_{\mathrm{b}}$ & $\begin{array}{l}\delta 0.90\left(\mathrm{t}, 3 \mathrm{H}, \text { term. } \mathrm{CH}_{3}\right) ; \delta 1.0-1.6(\mathrm{br} \mathrm{s} . ; 18 \mathrm{H}, \\
\underline{\mathrm{CH}}_{2} \text { chain); } \delta 2.34\left(\mathrm{t}, 2 \mathrm{H}, \mathrm{CH}_{2}-\mathrm{CONH}-\right) ; \delta 7.29(\mathrm{~d} \text {, } \\
1 \mathrm{Ha} \text {, one proton attached to sulfur atom in } \\
\text { thiazole ring }) ; \delta 7.53(\mathrm{~d}, 1 \mathrm{Hb}, \text { the } 2 \text { nd proton } \\
\text { beside the nitrogen atom in thiazole ring ) and } \delta \\
10.8 \text { (s., } 1 \mathrm{H} \text { for amide proton). }\end{array}$ & \multirow[t]{2}{*}{$\begin{array}{l}\text { Broad band at } 3170-3240 \mathrm{~cm}^{-1} \text { for }\left(v_{\mathrm{N}-\mathrm{H}}\right. \\
\text { of sec. amide), } 3020 \quad \mathrm{~cm}^{-1}\left(v_{\mathrm{C}-\mathrm{H}}\right. \\
\text { aromatic); 2980, 2920 and } 2870 \mathrm{~cm}^{-1} \\
\left(v_{\mathrm{C}-\mathrm{H}} \text { aliphatic); } 1670 \text { for }\left(v_{\mathrm{C}=\mathrm{O}} \text { of }\right.\right. \\
\text { amide }), \text { and } 1580,660 \mathrm{~cm}^{-1} \text { for }\left(v_{\mathrm{C}=\mathrm{N}},\right. \\
\left.v_{\mathrm{C}-\mathrm{S}}\right) \text { in thiazole ring respectively. }\end{array}$} \\
\hline $\mathbf{I I I}_{\mathrm{b}}$ & $\begin{array}{l}\delta 0.88\left(\mathrm{t}, 3 \mathrm{H} \text {, term. } \mathrm{CH}_{3}\right) ; \delta 1.1-1.6(\mathrm{br} \text { s.; } 18 \mathrm{H} \text {, } \\
\mathrm{CH}_{2} \text { chain); } \delta 2.34\left(\mathrm{t}, 2 \mathrm{H}, \quad \mathrm{CH}_{2}-\mathrm{CONH}-\right) ; \text { four } \\
\text { types of characteristics protons for pyridinium ring } \\
\text { at } \delta 7.20,8.02,8.15 \text {, and } 8.45 \text { and } \delta 10.20(\mathrm{~s} ., 1 \mathrm{H} \\
\text { for amide proton). }\end{array}$ & \\
\hline $\mathrm{IV} \mathrm{c}$ & $\begin{array}{l}\delta 0.88\left(\mathrm{t}, 3 \mathrm{H} \text {, term. } \mathrm{CH}_{3}\right) ; \delta 1.0-1.5(\mathrm{br} \mathrm{s} . ; 22 \mathrm{H}, \\
\frac{\mathrm{CH}_{2}}{2 \mathrm{H}} \text { chain); } \delta 2.36\left(\mathrm{t}, 2 \mathrm{H}, \mathrm{CH}_{2}-\mathrm{CONH}-\right) ; \delta 1.64(\mathrm{~m}, \\
3.2(\mathrm{~m} . ; 1 \mathrm{H} \text { for } \mathrm{CH}-\mathrm{OH}), \delta 3.4-3.7(\mathrm{~m} ., 2 \mathrm{H} \text { for }- \\
\underline{\mathrm{CH}}_{2}-\mathrm{SO}_{3}-\text { anion); } \delta 4.6(\mathrm{~s} ., 1 \mathrm{H} \text { for hydroxyl } \\
\text { group), and characteristics protons for thiazolium } \\
\text { ring at } \delta 7.60(\mathrm{~d} .2 \mathrm{H}) ; \delta 8.3(\mathrm{~d} ., 1 \mathrm{H} \text { in the ring) and } \\
\delta 9.3 \mathrm{ppm}(\mathrm{s} ., 1 \mathrm{H}) \text { for amide proton. }\end{array}$ & 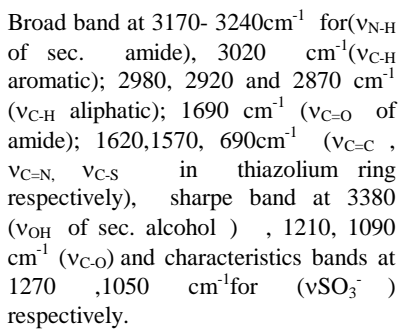 \\
\hline $\mathrm{V}_{\mathrm{e}}$ & $\begin{array}{l}\delta 0.90\left(\mathrm{t}, 3 \mathrm{H}, \text { term. } \mathrm{CH}_{3}\right) ; \delta 1.1-1.6(\mathrm{br} \mathrm{s} . ; 30 \mathrm{H}, \\
\underline{\mathrm{CH}}_{2} \text { chain); } \delta 2.4\left(\mathrm{t}, 2 \mathrm{H}, \mathrm{CH}_{2}-\mathrm{CONH}-\right) ; \delta 2.86(\mathrm{~s}, \\
\left.2 \mathrm{H}, \quad \text { OOC- }-\mathrm{CH}_{2}-\mathrm{N}+\right) \text { attached to thiazolium } \\
\text { ring, and characteristics protons for thiazolium } \\
\text { ring at } \delta 7.65(\mathrm{~d} .2 \mathrm{H}) ; \delta 8.2(\mathrm{~d} ., 1 \mathrm{H} \text { in the ring) and } \\
\delta 9.2 \mathrm{ppm}(\mathrm{s} ., 1 \mathrm{H}) \text { for amide proton. }\end{array}$ & \multirow{3}{*}{$\begin{array}{l}\text { Broad band at } 3170-3240 \mathrm{~cm}^{-1} \text { for }\left(v_{\mathrm{N}-\mathrm{H}}\right. \\
\text { of sec. amide }), 3020 \mathrm{~cm}^{-1}\left(v_{\mathrm{C}-\mathrm{H}}\right. \\
\text { aromatic }) ; 2980,2920 \text { and } 2870 \mathrm{~cm}^{-1} \\
\left(v_{\mathrm{C}-\mathrm{H}} \text { aliphatic); } 1650,1560 \mathrm{~cm}^{-1}\left(v_{\mathrm{C}=\mathrm{C}},\right.\right. \\
\left.v_{\mathrm{C}=\mathrm{N}} \text { in pyridinium ring }\right) \text { sharpe band } \\
\text { at } 3380\left(v_{\mathrm{OH}} \text { of sec. alcohol }\right), 1240, \\
1100 \mathrm{~cm}^{-1}\left(v_{\mathrm{C}-\mathrm{O}}\right) \text { and Characteristics } \\
\text { band at } 1270,1050 \mathrm{~cm}^{-1} \text { for }\left(\mathrm{vSO}_{3}^{-}\right) \\
\text {respectively. }\end{array}$} \\
\hline $\mathrm{VI}_{\mathrm{d}}$ & $\begin{array}{l}\left.\delta 0.87 \text { (t, } 3 \mathrm{H}, \text { term. } \mathrm{CH}_{3}\right) ; \delta 1.0-1.6(\mathrm{br} \text { s.; } 26 \mathrm{H} \text {, } \\
\underline{\mathrm{CH}}_{2} \text { chain); } \delta 2.34\left(\mathrm{t}, 2 \mathrm{H}, \mathrm{CH}_{2}-\mathrm{CONH}-\right) ; \delta 3,2( \\
\left.\mathrm{d} \text {., } 2 \mathrm{H} \text { for }-(\mathrm{HO})-\mathrm{CH}_{-}-\mathrm{CH}_{2}-\mathrm{N}+\right), \delta 3.1(\mathrm{~d} ., 2 \mathrm{H} \text { for } \\
-\mathrm{CH}_{2}-\mathrm{SO}_{3}-\text { anion); } \delta(\mathrm{s} ., 1 \mathrm{H} \text { for hydroxyl group), } \\
\text { four types of characteristics protons for pyridinium } \\
\text { ring at } \delta 9.2,8.50,9.0 \text { and } 9.40 \text { respectively and } \delta \\
10.4 \mathrm{ppm}(\mathrm{s} ., 1 \mathrm{H}) \text { for amide proton. }\end{array}$ & \\
\hline VIIa & $\begin{array}{l}\delta 0.87\left(\mathrm{t}, 3 \mathrm{H}, \text { term. } \mathrm{CH}_{3}\right) ; \delta 1.0-1.6(\text { br s.; } 14 \mathrm{H}, \\
\underline{\mathrm{CH}}_{2} \text { chain); } \delta 2.3\left(\mathrm{t}, 2 \mathrm{H}, \mathrm{CH}_{2}-\mathrm{CONH}-\right) ; \delta 2.90(\mathrm{~s}, \\
\left.2 \mathrm{H}, \text {, OOC- } \mathrm{CH}_{2}-\mathrm{N}+\right) \text { attached to pyridinium ring } \\
\text { and four types of characteristics protons for } \\
\text { pyridinium ring at } \delta 7.70,8.15,8.50 \text {, and } 9.05 \text { and } \\
\delta 9.80 \text { ( s., } 1 \mathrm{H} \text { for amide proton). }\end{array}$ & \\
\hline
\end{tabular}

\section{Surface properties}

Surface and interfacial tensions

The measured values of surface and interfacial tensions of the prepared surfactants are given in Table 4. The surface and interfacial tensions values increased with increasing the hydrophobicity in sulfobetaine and betaine products, respectively ${ }^{(4)}$. 
TABLE 4 . Surface properties of synthesized amphoteric containing thiazolium and pyridinium moiety .

\begin{tabular}{|c|c|c|c|c|c|c|c|c|}
\hline Comp. & $\begin{array}{c}\text { Surface } \\
\text { tension } \\
(\text { dyne/cm) } \\
0.1 \%\end{array}$ & $\begin{array}{c}\text { Interfacial } \\
\text { tension } \\
\text { (dyne/cm) } \\
0.1 \%\end{array}$ & $\begin{array}{c}\text { Kraft } \\
\text { point } \\
{ }^{\circ} \mathrm{C} \\
1 \%\end{array}$ & $\begin{array}{c}\text { Wetting } \\
\text { time } \\
\text { (sec.) } \\
0.1 \%\end{array}$ & 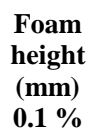 & $\begin{array}{l}\text { Stability to } \\
\text { (Acid) } \\
\text { min. : sec. }\end{array}$ & $\begin{array}{c}\text { hydrolysis } \\
\text { (Base) } \\
\text { min. : sec. }\end{array}$ & HLB \\
\hline IVa & 27.6321 & 7.3540 & 2 & 50 & 180 & $388: 00$ & $156: 23$ & 13.530 \\
\hline $\mathrm{IVb}$ & 27.9856 & 8.2354 & $<0$ & 49 & 260 & $396: 22$ & $159: 00$ & 12.627 \\
\hline IVc & 28.2081 & 8.42315 & $<0$ & 48 & 270 & $405: 45$ & $163: 08$ & 11.837 \\
\hline IVd & 29.3684 & 9.0032 & $<0$ & 51 & 280 & $422: 32$ & $169: 38$ & 11.141 \\
\hline IVe & 30.2529 & 9.2370 & $<0$ & 52 & 310 & $435: 05$ & $170: 50$ & 10.521 \\
\hline IVf & 30.6354 & 10.3213 & $<0$ & 56 & 320 & $446: 15$ & $172: 42$ & 10.564 \\
\hline IVg & 30.5236 & 9.9007 & $<0$ & 59 & 330 & $459: 33$ & $178: 50$ & 10.606 \\
\hline IVh & 31.3289 & 9.6573 & 12 & 64 & 360 & $490: 30$ & $186: 31$ & 10.564 \\
\hline IVi & 33.2238 & 10.6564 & 14 & 88 & 320 & $510: 15$ & $200: 22$ & 10.606 \\
\hline $\mathrm{Va}$ & 29.2584 & 7.4562 & 4 & 60 & 160 & $378: 36$ & $154: 32$ & 11.868 \\
\hline $\mathrm{Vb}$ & 29.5698 & 8.2564 & 2 & 65 & 180 & $381: 45$ & $156: 36$ & 10.887 \\
\hline $\mathrm{Vc}$ & 30.6498 & 9.2315 & $<0$ & 47 & 240 & $392: 00$ & $158: 00$ & 10.049 \\
\hline $\mathrm{Vd}$ & 31.7854 & 9.8976 & $<0$ & 54 & 250 & $413: 42$ & $163: 30$ & 9.346 \\
\hline $\mathrm{Ve}$ & 31.9876 & 9.0064 & 6 & 59 & 260 & $416: 40$ & $166: 33$ & 8.729 \\
\hline Vf & 32.1584 & 10.4633 & 5 & 64 & 280 & $432: 23$ & $169: 40$ & 8.771 \\
\hline $\mathrm{Vg}$ & 34.3597 & 10.9007 & 3 & 68 & 290 & $446: 38$ & $176: 32$ & 8.812 \\
\hline $\mathrm{Vh}$ & 35.2982 & 10.6573 & 17 & 72 & 300 & $480: 24$ & $183: 12$ & 8.771 \\
\hline IVi & 36.8760 & 12.7654 & 15 & 93 & 290 & $590: 41$ & $199: 22$ & 8.812 \\
\hline VIa & 29.4323 & 7.0321 & 5 & 55 & 130 & $388: 00$ & $156: 23$ & 13.415 \\
\hline $\mathrm{VIb}$ & 29.8737 & 7.9758 & 3 & 59 & 140 & $396: 22$ & $159: 00$ & 12.507 \\
\hline VIc & 31.2589 & 8.2315 & $<0$ & 49 & 170 & $405: 45$ & $163: 08$ & 11.714 \\
\hline VId & 32.6472 & 9.1254 & $<0$ & 56 & 200 & $422: 32$ & $169: 38$ & 11.072 \\
\hline VIe & 33.3526 & 9.9872 & $<0$ & 67 & 210 & $435: 05$ & $170: 50$ & 10.396 \\
\hline VIf & 34.6857 & 10.0214 & $<0$ & 68 & 220 & $446: 15$ & $172: 42$ & 10.430 \\
\hline VIg & 34.9876 & 10.8763 & $<0$ & 70 & 230 & $459: 33$ & $178: 50$ & 10.472 \\
\hline VIh & 35.6422 & 11.0125 & 14 & 79 & 200 & $490: 30$ & $186: 31$ & 10.430 \\
\hline VIi & 37.4322 & 13.6564 & 12 & 110 & 380 & $550: 30$ & $230: 11$ & 10.472 \\
\hline VIIa & 30.3224 & 9.0321 & 8 & 60 & 120 & $375: 36$ & $159: 55$ & 11.693 \\
\hline VIIb & 30.3698 & 9.6864 & 6 & 65 & 130 & $386: 29$ & $160: 46$ & 11.713 \\
\hline VIIc & 31.9876 & 10.2315 & 1 & 47 & 160 & $390: 38$ & $163: 27$ & 9.883 \\
\hline VIId & 32.2620 & 10.5314 & $<0$ & 54 & 180 & $410: 24$ & $165: 07$ & 9.714 \\
\hline VIIe & 33.6974 & 10.0022 & $<0$ & 59 & 170 & $415: 42$ & $166: 32$ & 8.559 \\
\hline VIIf & 34.6983 & 11.4664 & $<0$ & 64 & 200 & $418: 03$ & $178: 44$ & 8.600 \\
\hline VIIg & 35.3597 & 12.9007 & $<0$ & 68 & 180 & $421: 59$ & $186: 39$ & 8.642 \\
\hline VIIh & 37.0002 & 13.6573 & 9 & 72 & 190 & $469: 06$ & $191: 33$ & 8.600 \\
\hline VIIi & 38.3256 & 13.4321 & 8 & 95 & 290 & $480: 52$ & $210: 00$ & 8.642 \\
\hline
\end{tabular}

Error of measurements was

Surface and interfacial tensions $= \pm 0.1 \mathrm{dyne} / \mathrm{cm}$

Kraft point $= \pm 1{ }^{\circ} \mathrm{C}-\mathrm{HLB}= \pm 0.2$

Foam height $= \pm 2 \mathrm{~mm}$

Wetting time $= \pm 1 \mathrm{Sec}$

Stability to hydrolysis $= \pm 1 \mathrm{~min}$

Egypt. J. Chem. 54, No. 2 (2011) 
TABLE 5 . Emulsification power of the synthesized amphoteric surfactants .

\begin{tabular}{|c|c|c|c|c|c|c|c|c|c|c|}
\hline \multirow{3}{*}{$\begin{array}{c}\text { Compound } \\
\text { No. } \\
\text { IVa }\end{array}$} & \multicolumn{2}{|c|}{$\begin{array}{c}t=0.0 \\
\min \end{array}$} & \multicolumn{2}{|c|}{$\begin{array}{c}\mathrm{t}=5.0 \\
\mathrm{~min}\end{array}$} & \multicolumn{2}{|c|}{$\begin{array}{c}\mathrm{t}=\mathbf{1 0 . 0} \\
\min \end{array}$} & \multicolumn{2}{|c|}{$\mathrm{t}=20.0 \mathrm{~min}$} & \multicolumn{2}{|c|}{$t=30.0 \mathrm{~min}$} \\
\hline & $\mathrm{e}$ & 5 & $\mathrm{e}$ & 4.0 & $\mathrm{e}$ & 4.0 & $\mathrm{e}$ & 3.0 & $\mathrm{e}$ & 1.5 \\
\hline & $\mathrm{o}$ & 0 & $\mathrm{o}$ & 1.0 & $\mathrm{o}$ & 1.0 & $\mathrm{o}$ & 2.0 & o & 3.5 \\
\hline \multirow[t]{2}{*}{ IVb } & $\mathrm{e}$ & 5 & $\mathrm{e}$ & 4.5 & $\mathrm{e}$ & 4.0 & $\mathrm{e}$ & 3.0 & $\mathrm{e}$ & 1.5 \\
\hline & $\mathrm{o}$ & 0 & $\mathrm{o}$ & 0.5 & $\mathrm{o}$ & 1.0 & $\mathrm{o}$ & 2.0 & o & 3.5 \\
\hline \multirow[t]{2}{*}{ IVc } & $\mathrm{e}$ & 5 & $\mathrm{e}$ & 5.0 & $\mathrm{e}$ & 3.5 & $\mathrm{e}$ & 3.5 & $\mathrm{e}$ & 2.0 \\
\hline & o & 0 & o & 0.0 & $\mathrm{o}$ & 1.5 & $\mathrm{o}$ & 1.5 & o & 3.0 \\
\hline \multirow[t]{2}{*}{ IVd } & $\mathrm{e}$ & 5 & $\mathrm{e}$ & 4.5 & $\mathrm{e}$ & 4.0 & $\mathrm{e}$ & 3.5 & $\mathrm{e}$ & 2.5 \\
\hline & $\mathrm{o}$ & 0 & $\mathrm{o}$ & 0.5 & $\mathrm{o}$ & 1.0 & $\mathrm{o}$ & 1.5 & $\mathrm{o}$ & 2.5 \\
\hline \multirow[t]{2}{*}{ IVe } & $\mathrm{e}$ & 5 & $\mathrm{e}$ & 4.5 & $\mathrm{e}$ & 4.0 & $\mathrm{e}$ & 3.5 & $\mathrm{e}$ & 3.0 \\
\hline & $\mathrm{o}$ & 0 & $\mathrm{o}$ & 0.5 & $\mathrm{o}$ & 1.0 & o & 1.5 & $\mathrm{o}$ & 2.0 \\
\hline \multirow[t]{2}{*}{ IVf } & $\mathrm{e}$ & 5 & $\mathrm{e}$ & 4.0 & $\mathrm{e}$ & 3.5 & $\mathrm{e}$ & 3.0 & $\mathrm{e}$ & 2.5 \\
\hline & o & 0 & o & 1.0 & o & 1.5 & 0 & 2.0 & o & 2.5 \\
\hline \multirow[t]{2}{*}{ IVg } & $\mathrm{e}$ & 5 & $\mathrm{e}$ & 4.5 & $\mathrm{e}$ & 4.0 & $\mathrm{e}$ & 3.5 & $\mathrm{e}$ & 2.5 \\
\hline & o & 0 & o & 0.5 & o & 1.0 & o & 1.5 & o & 2.5 \\
\hline \multirow[t]{2}{*}{ IVh } & $\mathrm{e}$ & 5 & $\mathrm{e}$ & 5.0 & $\mathrm{e}$ & 4.5 & $\overline{\mathrm{e}}$ & 4.0 & $\mathrm{e}$ & 3.0 \\
\hline & $\mathrm{o}$ & 0 & 0 & 0.0 & $\mathrm{o}$ & 0.5 & $\mathrm{o}$ & 1.0 & $\mathrm{o}$ & 2.0 \\
\hline \multirow[t]{2}{*}{ IVi } & $\mathrm{e}$ & 5 & $\mathrm{e}$ & 5.0 & $\mathrm{e}$ & 4.5 & $\mathrm{e}$ & 4.0 & $\mathrm{e}$ & 3.5 \\
\hline & o & 0 & o & 0.0 & o & 0.5 & o & 1.0 & o & 1.5 \\
\hline \multirow[t]{2}{*}{$\mathbf{V a}$} & $\mathrm{e}$ & 5 & $\mathrm{e}$ & 4.0 & $\overline{\mathrm{e}}$ & 3.0 & $\mathrm{e}$ & 2.5 & $\mathrm{e}$ & 1.0 \\
\hline & o & 0 & o & 1.0 & o & 2.0 & o & 2.5 & o & 4.0 \\
\hline \multirow[t]{2}{*}{$\mathbf{V b}$} & $\mathrm{e}$ & 5 & $\mathrm{e}$ & 4.5 & $\mathrm{e}$ & 3.5 & $\mathrm{e}$ & 3.0 & $\mathrm{e}$ & 1.5 \\
\hline & o & 0 & o & 0.5 & o & 1.5 & o & 2.0 & o & 3.5 \\
\hline \multirow[t]{2}{*}{$\overline{V c}$} & $\mathrm{e}$ & 5 & $\overline{\mathrm{e}}$ & 4.5 & $\overline{\mathrm{e}}$ & 4.0 & $\overline{\mathrm{e}}$ & 3.5 & $\overline{\mathrm{e}}$ & 2.0 \\
\hline & o & 0 & $\mathrm{O}$ & 0.0 & o & 1.0 & O & 1.5 & o & 3.0 \\
\hline \multirow[t]{2}{*}{$\overline{V d}$} & $\mathrm{e}$ & 5 & $\overline{\mathrm{e}}$ & 4.5 & $\overline{\mathrm{e}}$ & 4.0 & $\overline{\mathrm{e}}$ & 3.5 & $\overline{\mathrm{e}}$ & 2.0 \\
\hline & o & 0 & $\mathrm{o}$ & 0.5 & o & 1.0 & $\mathrm{o}$ & 1.5 & o & 3.0 \\
\hline Ve & $\mathrm{e}$ & 5 & $\mathrm{e}$ & 4.5 & $\mathrm{e}$ & 4.0 & $\mathrm{e}$ & 3.5 & $\mathrm{e}$ & 2.5 \\
\hline & o & 0 & o & 0.5 & o & 1.0 & o & 1.5 & o & 2.5 \\
\hline Vf & $\mathrm{e}$ & 5 & $\mathrm{e}$ & 4.0 & $\mathrm{e}$ & 3.5 & $\mathrm{e}$ & 3.0 & $\mathrm{e}$ & 2.5 \\
\hline & o & 0 & o & 1.0 & o & 1.5 & o & 2.0 & o & 2.5 \\
\hline $\mathrm{Vg}$ & $\mathrm{e}$ & 5 & $\mathrm{e}$ & 4.5 & $\mathrm{e}$ & 4.0 & $\bar{e}$ & 3.5 & $\mathrm{e}$ & 2.0 \\
\hline & o & 0 & o & 0.5 & o & 1.0 & o & 1.5 & o & 3.0 \\
\hline Vh & $\mathrm{e}$ & 5 & $\mathrm{e}$ & 5.0 & $\mathrm{e}$ & 4.5 & $\mathrm{e}$ & 4.0 & $\mathrm{e}$ & 2.5 \\
\hline & $\mathrm{o}$ & 0 & $\mathrm{o}$ & 0.0 & $\mathrm{o}$ & 0.5 & $\mathrm{O}$ & 1.0 & 0 & 2.5 \\
\hline $\mathbf{V i}$ & $\mathrm{e}$ & 5 & $\mathrm{e}$ & 5.0 & $\mathrm{e}$ & 4.5 & $\mathrm{e}$ & 4.0 & $\mathrm{e}$ & 3.5 \\
\hline & o & 0 & o & 0.0 & o & 0.5 & o & 1.0 & o & 1.5 \\
\hline VIa & $\mathrm{e}$ & 5 & $\mathrm{e}$ & 4.0 & $\overline{\mathrm{e}}$ & 3.5 & $\bar{e}$ & 3.0 & $\mathrm{e}$ & 2.0 \\
\hline & o & 0 & o & 1.0 & o & 1.5 & o & 2.0 & o & 3.0 \\
\hline VIb & $\mathrm{e}$ & 5 & $\mathrm{e}$ & 4.0 & $\mathrm{e}$ & 3.0 & $\mathrm{e}$ & 2.5 & $\mathrm{e}$ & 2.0 \\
\hline & o & 0 & o & 1.0 & o & 2.0 & o & 2.5 & o & 3.0 \\
\hline VIc & $\mathrm{e}$ & 5 & $\overline{\mathrm{e}}$ & 4.0 & $\overline{\mathrm{e}}$ & 3.5 & $\overline{\mathrm{e}}$ & 3.0 & $\overline{\mathrm{e}}$ & 2.5 \\
\hline & o & 0 & o & 1.0 & o & 1.5 & o & 2.0 & o & 2.5 \\
\hline VId & $\mathrm{e}$ & 5 & $\mathrm{e}$ & 4.5 & $\overline{\mathrm{e}}$ & 4.0 & $\overline{\mathrm{e}}$ & 3.5 & $\overline{\mathrm{e}}$ & 2.5 \\
\hline & o & 0 & o & 0.5 & o & 1.0 & $\mathrm{O}$ & 1.5 & o & 2.5 \\
\hline VIe & $\mathrm{e}$ & 5 & $\mathrm{e}$ & 4.5 & $\mathrm{e}$ & 4.0 & $\mathrm{e}$ & 3.5 & $\mathrm{e}$ & 3.0 \\
\hline & o & 0 & o & 0.5 & o & 1.0 & o & 1.5 & o & 2.0 \\
\hline VIf & $\mathrm{e}$ & 5 & $\mathrm{e}$ & 4.0 & $\mathrm{e}$ & 3.5 & $\mathrm{e}$ & 3.0 & $\mathrm{e}$ & 2.5 \\
\hline & o & 0 & o & 1.0 & o & 1.5 & o & 2.0 & o & 2.5 \\
\hline VIg & $\mathrm{e}$ & 5 & $\mathrm{e}$ & 4.5 & $\mathrm{e}$ & 4.0 & $\mathrm{e}$ & 3.0 & $\mathrm{e}$ & 3.0 \\
\hline & o & 0 & o & 0.5 & o & 1.0 & o & 2.0 & o & 2.0 \\
\hline
\end{tabular}




\begin{tabular}{|c|c|c|c|c|c|c|c|c|c|c|}
\hline \multirow{2}{*}{$\begin{array}{c}\text { Compound } \\
\text { No } \\
\text { VIh }\end{array}$} & \multirow{2}{*}{$\begin{array}{r}\mathrm{t}= \\
\mathrm{e}\end{array}$} & \multirow{2}{*}{$\begin{array}{c}0.0 \mathrm{~min} \\
5\end{array}$} & \multicolumn{2}{|c|}{$\mathrm{t}=5.0 \mathrm{~min}$} & \multicolumn{2}{|c|}{$t=10.0 \mathrm{~min}$} & \multicolumn{2}{|c|}{$\mathrm{t}=20.0 \mathrm{~min}$} & \multicolumn{2}{|c|}{$\mathrm{t}=30.0 \mathrm{~min}$} \\
\hline & & & $\mathrm{e}$ & 5.0 & $\mathrm{e}$ & 4.5 & $\mathrm{e}$ & 4.0 & $\mathrm{e}$ & 3.5 \\
\hline & o & 0 & o & 0.0 & o & 0.5 & o & 1.0 & o & 1.5 \\
\hline \multirow[t]{2}{*}{ VIi } & $\mathrm{e}$ & 5 & $\mathrm{e}$ & 5.0 & $\mathrm{e}$ & 4.5 & $\mathrm{e}$ & 4.0 & $\mathrm{e}$ & 3.5 \\
\hline & o & 0 & o & 0.0 & o & 0.5 & o & 1.0 & o & 1.5 \\
\hline \multirow[t]{2}{*}{ VIIa } & $\overline{\mathrm{e}}$ & 5 & $\overline{\mathrm{e}}$ & 4.0 & $\overline{\mathrm{e}}$ & 3.5 & $\overline{\mathrm{e}}$ & 3.0 & $\overline{\mathrm{e}}$ & 2.5 \\
\hline & o & 0 & o & 1.0 & o & 1.5 & $\mathrm{o}$ & 2.0 & o & 2.5 \\
\hline \multirow[t]{2}{*}{ VIII } & $\overline{\mathrm{e}}$ & 5 & $\mathrm{e}$ & 4.0 & $\mathrm{e}$ & 3.0 & $\mathrm{e}$ & 2.5 & $\mathrm{e}$ & 2.0 \\
\hline & o & 0 & o & 1.0 & $\mathrm{o}$ & 2.0 & $\mathrm{o}$ & 2.5 & o & 3.0 \\
\hline \multirow{2}{*}{ VIIc } & $\overline{\mathrm{e}}$ & 5 & $\overline{\mathrm{e}}$ & 4.0 & $\overline{\mathrm{e}}$ & 3.5 & $\overline{\mathrm{e}}$ & 3.0 & $\overline{\mathrm{e}}$ & 2.5 \\
\hline & $\mathrm{o}$ & 0 & o & 1.0 & o & 1.5 & $\mathrm{o}$ & 2.0 & o & 2.5 \\
\hline \multirow[t]{2}{*}{ VIId } & $\mathrm{e}$ & 5 & $\mathrm{e}$ & 4.5 & $\mathrm{e}$ & 4.0 & $\mathrm{e}$ & 3.0 & $\mathrm{e}$ & 2.5 \\
\hline & o & 0 & o & 0.5 & o & 1.0 & o & 2.0 & o & 2.5 \\
\hline \multirow[t]{2}{*}{ VIIe } & $\mathrm{e}$ & 5 & $\mathrm{e}$ & 4.5 & $\mathrm{e}$ & 4.0 & $\mathrm{e}$ & 3.5 & $\mathrm{e}$ & 3.0 \\
\hline & $\mathrm{o}$ & 0 & o & 0.5 & o & 1.0 & o & 1.5 & o & 2.0 \\
\hline \multirow[t]{2}{*}{ VIIf } & $\mathrm{e}$ & 5 & $\overline{\mathrm{e}}$ & 4.0 & $\overline{\mathrm{e}}$ & 3.5 & $\overline{\mathrm{e}}$ & 3.0 & $\mathrm{e}$ & 2.5 \\
\hline & o & 0 & o & 1.0 & $\mathrm{o}$ & 1.5 & $\mathrm{o}$ & 2.0 & o & 2.5 \\
\hline \multirow[t]{2}{*}{ VIIg } & $\overline{\mathrm{e}}$ & 5 & $\mathrm{e}$ & 4.5 & $\bar{e}$ & 4.0 & $\overline{\mathrm{e}}$ & 3.0 & $\overline{\mathrm{e}}$ & 2.5 \\
\hline & o & 0 & $\mathrm{o}$ & 0.5 & o & 1.0 & $\mathrm{O}$ & 2.0 & o & 2.5 \\
\hline \multirow[t]{2}{*}{ VIIh } & $\mathrm{e}$ & 5 & $\mathrm{e}$ & 5.0 & $\mathrm{e}$ & 3.5 & $\mathrm{e}$ & 4.0 & $\mathrm{e}$ & 3.5 \\
\hline & o & 0 & o & 0.0 & o & 1.5 & o & 1.0 & $\mathrm{o}$ & 1.5 \\
\hline \multirow[t]{2}{*}{ VIIi } & $\overline{\mathrm{e}}$ & 5 & $\overline{\mathrm{e}}$ & 5.0 & $\overline{\mathrm{e}}$ & 4.5 & $\overline{\mathrm{e}}$ & 4.0 & $\overline{\mathrm{e}}$ & 3.5 \\
\hline & o & 0 & o & 0.0 & o & 0.5 & o & 1.0 & o & 1.5 \\
\hline
\end{tabular}

\section{Kraft Point}

The recorded data in Table 4, showed that the surfactants with thiazolium moiety with sulfobetaines of hydrophilic unite satisfy lower values of Kraft point $\left(\mathrm{T}_{\mathrm{Kp}}\right)$. This, might lead to wide uses in industrial applications ${ }^{(4)}$.

\section{Wetting time}

The wetting properties of a surfactant is one of its most important surface properties. For example, in laundry cleaning or textile processing, the wetting of surfactants may accelerate the diffusion or penetration of alkali chemicals and dyes into fibers and improve the detergency. From the measured data of the wetting time for the prepared surfactants are illustrated in Table 4. Wetting properties for sulfo-betaines with thiazolium moiety for grape seed oil IV $_{i}$ recorded good result than other prepared compounds, while the effect of the hydrophobic moiety is negligible ${ }^{(23)}$.

\section{Foaming height}

Low-foaming tendency of surfactants is recently considered as an important property in some applications such as dyeing auxiliaries in modern textile dyeing industry. From the data recorded in Table 3 surfactants $\mathrm{IV}_{\mathrm{i}}, \mathrm{V}_{\mathrm{i}}, \mathrm{VI}_{\mathrm{i}}$ and $\mathrm{VII}_{\mathrm{i}}$ for mixed fatty derivatives of gawafa fat and grape oil reveal higher foam than all prepared compounds. The relative low-foaming properties of the amphoteric surfactants( amido-betaines) containing pyridinium moiety are recorded for VIIa-g.

Egypt. J. Chem. 54, No. 2 (2011) 
Stability to hydrolysis

From the data recorded in Table 4 stability of the synthesized Amphoteric surfactants. It can be seen that all the prepared compound have higher stability in acidic media than in basic medium, this may be due the presence of nitrogen atoms which coordinated with the acid forming more cationic center ${ }^{(24-25)}$.

\section{Emulsification stability}

It was reported that, better emulsifying properties were obtained with derivatives containing pyridinium moiety incorporated with acetate group into their structure ${ }^{(23)}$. From the data given in Table 5, the prepared compounds exhibit an excellent degree to form emulsions either $\mathrm{O} / \mathrm{W}$ or $\mathrm{W} / \mathrm{O}$ for those with high percent of hydrophobicity with pyridinum moiety.

\section{Hydrophilic-lipophilic balance (HLB),}

Of a surfactant, one of the most widely used indicators of it suitability for a given application is a measure of a surfactant partitioning tendency between oil and water. Since Griffin first introduced this definition in $1949^{(26)}$. From data recorded in Table 5, HLB values decrease with increase the number of carbon atoms in alkyl chain. On other hand, HLB values for sulfo-thiazolium amphoteric surfactant revealed higher values than other prepared compounds. Also, amphoteric surfactants prepared from grape oil and Juagafa fat given hlb values 8.6-10.6. These values indicate that the synthesized amphoteric surfactants can be used as (W/O (water in oil) emulsifier or oil in water emulsion).

\section{Biodegradability}

After use, all surfactants used in laundry detergents, cleaning agents, and dyeing auxiliaries are passed quantitatively into waste water. Because of this fact, the constant input of surfactants into the environment requires a particular ecological characterization of this class of compounds. An excellent review of surfactant biodegradability points out that biodegradability increases with increasing linearity of the hydrophobic group and is reduced, for isomeric materials, by branching in that group and in the presence of aromatic ring ${ }^{(27,28)}$. Biodegradability is deterred and degradation is slowed as steric hindrance increases ${ }^{(29-30)}$. Figures 1 and 2 revealed the degradation of the amphoteric surfactants containing the pyridinium moiety $\left(\mathrm{VI}_{\mathrm{a}}\right.$ and $\left.\mathrm{VII}_{\mathrm{a}}\right)$ more than the surfactant containing thiazolium moiety $\left(\mathrm{IV}_{\mathrm{b}}\right.$ and $\left.\mathrm{IV}_{\mathrm{e}}\right)$.

\section{Biological activity}

Antimicrobial activity of the prepared compounds was tested via a modification of the cup- plate method ${ }^{(31)}$. All the prepared surfactants were screened for their bactericidal activities against (Bacillus sublitis and Escherichia coil) and their antifungal activity against (Aspergillus niger and Candida albicam). The results are listed in Table 6. It is apparent from that some of the synthesized compounds showed antibacterial activity. However, concerning the activity against Gram positive bacteria compounds $I_{\mathrm{b}}, \mathrm{IV}_{\mathrm{f}}, \mathrm{IV}_{\mathrm{h}}$ and $\mathrm{IV}_{\mathrm{i}}$ exhibit good activity, where as compounds $\mathrm{V}_{\mathrm{b}}$, $\mathrm{VII}_{\mathrm{d}}$, and $\mathrm{VII}_{\mathrm{i}}$ showed moderate activity. 


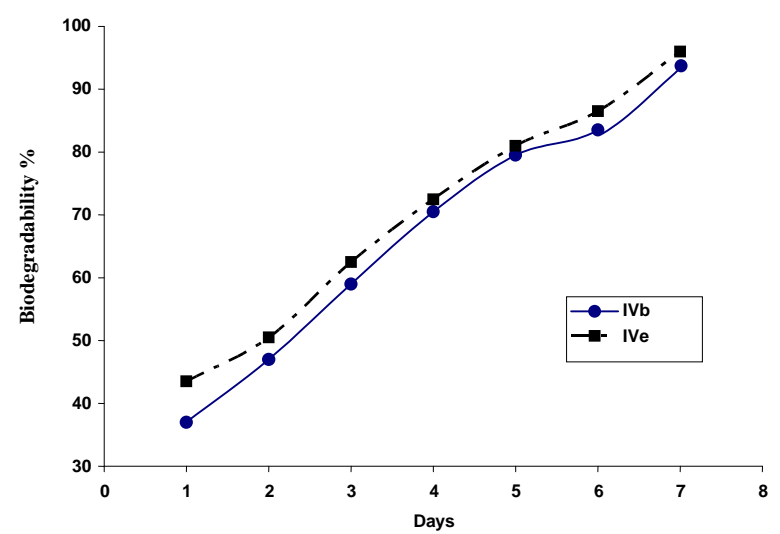

Fig. 1. Biodegradability \% of amphoteric surfactant containing thiazolium moiety.

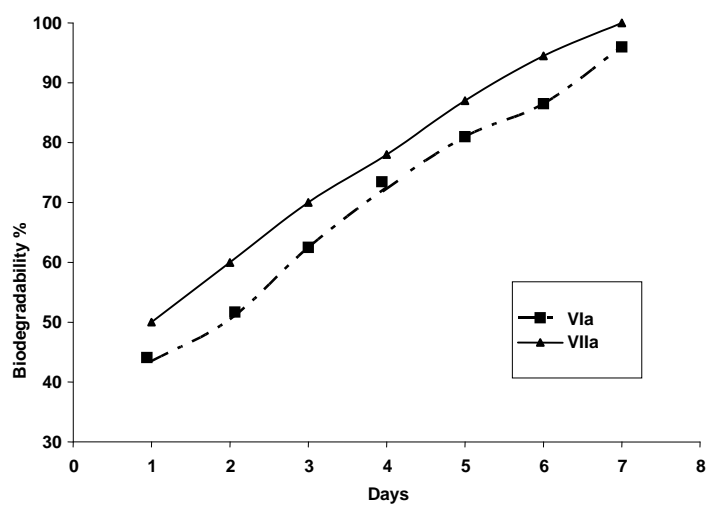

Fig. 2. Biodegradability \% of amphoteric surfactant containing pyridinium moiety.

\section{Conclusion}

It can be concluded that all prepared amphoteric surfactants exhibited antibacterial and antifungal properties as well as emulsifier properties especially for compound from Grape seed oil and Gawafa fat . Therefore, their potential use in a non edible media such as insecticides or pesticides as well as in the manufacturing of drugs, cosmetics, antibacterial and/or antifungal is recommended.

Acknowledgments: Origin of cultures: Botany Department, Faculty of Science, Benha University, Egypt.

Egypt. J. Chem. 54, No. 2 (2011) 
TABLE 6. Biological activity of some prepared amphoteric surfactants.

\begin{tabular}{|c|c|c|c|c|}
\hline \multirow{2}{*}{$\begin{array}{l}\text { Compound } \\
\text { number }\end{array}$} & \multicolumn{2}{|c|}{ Antibacterial } & \multicolumn{2}{|c|}{ Antifungal } \\
\hline & Bacillus Subti & Escherichia C & Aspergillus nig & Candida albis \\
\hline$I_{V_{b}}$ & ++ & + & +++ & +++ \\
\hline $\mathbf{I V}_{\mathrm{f}}$ & +++ & ++ & ++ & ++ \\
\hline $\mathbf{I V} \mathbf{V}_{\mathbf{h}}$ & ++ & + & ++ & ++ \\
\hline$I_{V_{i}}$ & + & + & +++ & +++ \\
\hline $\mathbf{V}_{\mathbf{f}}$ & ++ & ++ & + & ++ \\
\hline$V_{h}$ & + & + & - & - \\
\hline $\mathbf{V}_{\mathbf{i}}$ & ++ & + & - & - \\
\hline $\mathbf{I V}_{\mathrm{b}}$ & ++ & ++ & + & + \\
\hline$I_{V_{f}}$ & ++ & ++ & + & ++ \\
\hline$V I_{b}$ & ++ & ++ & + & ++ \\
\hline VII $_{f}$ & ++ & + & - & - \\
\hline $\mathbf{V I I}_{h}$ & ++ & + & - & - \\
\hline
\end{tabular}

$(+++)$ Very strong inhibition, $(++)$ strong inhibition, $(+)$ moderate inhibition

\section{References}

1. El-Dougdoug, W.I.A. and Ahmed, M.H.M., J. Oil, Soap and Cosmetics, 50 (1), 25-29 (2001).

2. El-Sawy, A. A., Essawy, S. A., Hebash, K. A. H. and El - Dougdoug , W.I.A., Preparation of epoxy fatty derivatives from industrial wastes. Seifen -Ole-FetteWachse, 112(2), 63-65(1991).

3. El-Dougdoug, W. I. A., Grasas Y Aceites, 50 (5), 385-391(1999).

4. El-Dougdoug, W.I.A. and Ahmed, M.H.M., Synthesis and surface active properties of Gawafa fats based amphoteric surfactants. J. Oil Soap Cosmetics, 53(2), 63-67(2004) .

5. Abou Rayan, M.A., Abdel-Nabey, A.A., Abou Samaha O. R. and Mohamed, M.K., Characteristics and composition of grape seed oil. Alex. J. Agric. Res. 43(1), 67-79(1998).

6. Gawish, S.M., Hazzaa, A. A. B. and El-Din Geberil, J. Am. Oil Chem. Soc. 55, 745-747(1978).

7. Parris, N., Wiel, J.K. and Linfield, W.M., J. Am. Oil Chem. Soc. 53, 60-63(1979).

8. El-Sayed, R., Surface active properties and biological activity of novel nonionic surfactants containing pyrimidines and related nitrogen heterocyclic ring systems. Grasas Y Aceites, 59 (2), 110-120(2008).

9. Xu , L.Z., Jian, F.F., Shi, J.G. and Li, L., Synthesis, structure and biological activities of novel triazole compounds containing 4,6-dimethyl-pyrimidine-2-thio group. $J$. Chinese Chem. Soc. 22, 1308-1312(2004).

10. Eman, A.E. and Mohamed, A.K., 2-Cyanoacetamide in the synthesis of heterocyclic compounds: Synthesis of new polysubstituted pyrazole, pyridine and pyramimidine derivatives. J. Chinese Chem. Soc. 51, 779-784(2004). 
11. Ahmed, Maisa, I. A., A. G., Galal, M.E. and Soliman, M.M., Synthesis of new furo[2,3-d]pyrimidine and pyrimido[4 $5: 5,5]$ furo[2,3-d] pyrimidines. J. Chinese Chem. Soc. 51, 1357-1366 (2004).

12. El-Sawy, A. A., Grasas, Y. Aceites, 40, 382-384 (1989).

13. Youngs, C.G., Epp, A., Craig, B.M. and Sallans, H.R., Preparation of long chain fatty acid chloride. J. Am. Oil Chem. 34, 107(1955).

14. Findly, A., Practical Physical Chemistry: $6^{\text {th }}$ ed., London: Longmans, London: p.1040 (1963).

15. Weil,J.K., Smith, F.D., Stirton, A. J. and Bristlin,R. G. Jr., J. Am. Oil Chem. Soc. 40, 538( 1963).

16. Draves, C. Z. and Clarkes, R., J. Am. Dye Stuff Repoter, 20, 538(1931).

17. Ross, J. and Miles, G.D., Apparatus for comparison of foaming of soaps and detergent. Oil and Soap, 18, 99 ( 1941).

18. Takeshi , H., Bull. Chem. Soc. LB, 2236. (Japan). (1970).

19. El-Sukkary, M.M.A., El-Sawy, A.A. and El-Dib, F., Hungarian J. Industr. Chem. 15,417(1987) .

20. Xiaowen, G., Zongming, R. and Xugen, Y., Calculation of hydrophile-lipophile balance for polyethoxylated surfactants by group contribution method. J. Colloid and Interface Science, 298, 441-450(2006).

21. Griffin,W.C., Emulsification. In: Cosmetics Science and Technology, M.S. Balsam and E. Sagarin, (Ed.), Wiley, New York, Vol. 3, 573-629(1974).

22. Eter, E.T., Richard, R. E. and Darid, A., Biodegradable surfactants derived from corn starch. J. Am. Oil Chem. Soc. 51, 486- 494 (1974).

23. Falbe, J., Surfactants for Consumer. Chapter 4, Springer-Verlager, Heidelberg, ISBN 0-387-17019-7, 139. (U.S) (1986).

24. Eissa, A. M. F., Mahmoud, A.A. and El- Sawy, A. A., 45, 306-308(1993).

25. El-Dougdoug, W. I. A., J. Dispersion Science and Technology, 31, 1298-1042 (2010).

26. Griffin, W. C., J. Soc. Cosmet. Chem. 1, 311(1949).

27. Szymanski, A., Wyras, B., Zbigniew, S., Jaroszynski, T. and Lukaszeqski, Z., Water Res. 34, 410(2000).

28. Chen, L., Hu, Z. and Zhu, H. Synthesis of cleavable aryl sulfonates and a study of their surface activity. 11, 97(2008)

29. Daniel Prats, Carmen López, Diana Vallejo, Pedro Varó, and Víctor M. León, $J$. Surf. and Deterg. 9, 69 (2006).

30. Yasin, S. A. and El-Dougdoug, W.I.A., J. Dispersion Science and Technology, 31, 1033-1042 (2010).

Egypt. J. Chem. 54, No. 2 (2011) 
31. Marei, A., El-Sukkary, M.M.A., El-Dib, F.I. and El-Sayed, H., Hungarian Journal of Industrial Chemistry, 9, 427 - 440( 1981).

(Received 25/7/2011

accepted 15/8/2011)

\section{مواد ذات نشاط سطحي أمفوتيرية ذات حلقات غير متجانسة من \\ المخلفات الصناعية}

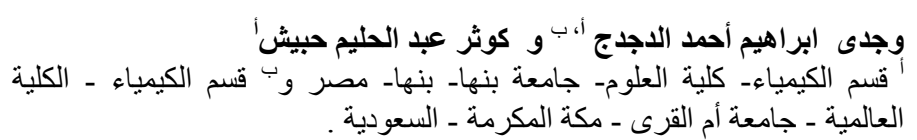

يتناول هذا البحث تشبيد بعض المركبات ذات النشاط السطحي الأمفوتيرية و التي

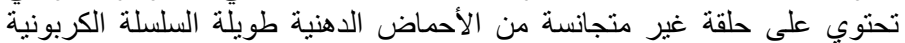
وكذلك مخلوط الأحماض المحضر من دهن بذور الجو افة و العنب المحلي).

$$
\text { ويتم هذا علي النحو التالي:- }
$$

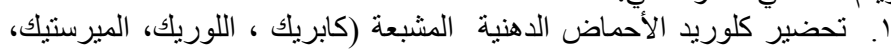

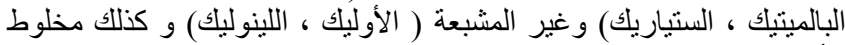

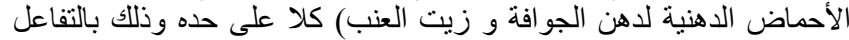

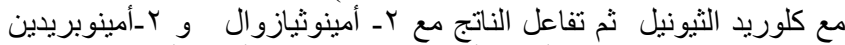

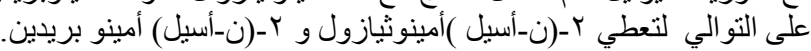

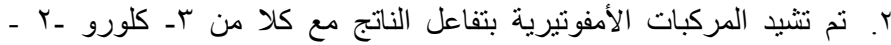

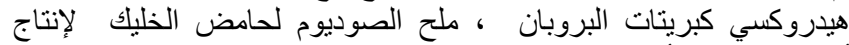
أميدوسلفوبيتين، أميدوبينين.

r. . وقد تم التعرف و إثبات التركيب الكيميائي لتلك المركبات بالتحليل العنصري

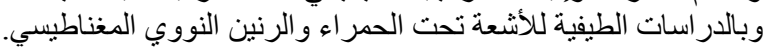

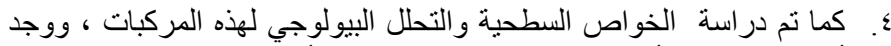

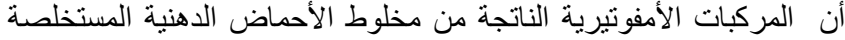

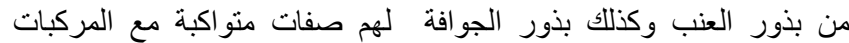

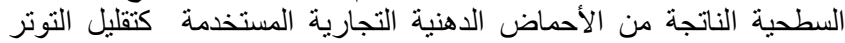

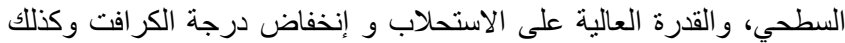

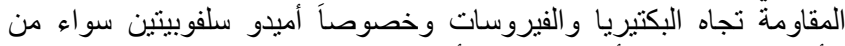

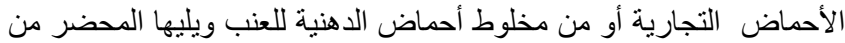

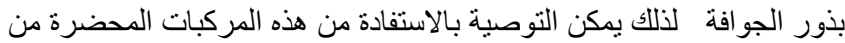

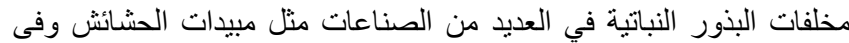

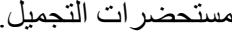

